\title{
Beiträge zur Kenntnis der sogenannten „Schwimmhölzer“.
}

Von Elisabeth Ewald.

Unter der Bezeichnung "Schwimmhölzer" sind in der Literatur eine Reihe von Hölzern beschrieben worden, die sämtlich auffallend leicht sind und auch im anatomischen Bau weitgehende Übereinstimmung zeigen. Einige derselben sind von Wiesner unter die „Korkhölzer“ eingereiht worden. Gegen die von de Bary eingeführte und von Strasburger aufgenommene Bezeichnung „Schwimmholz“ wendet Groebel ein, daß das "Schwimmholz" ja nichts mit dem Schwimmen der Pflanze zu tun habe; denn es handle sich nur um einen Ausnahmefall, wenn sie sich an der Bildung "schwimmender Inseln" beteilige.

Wiesners Bezeichnung „Korkholz" aber übersieht die Entwicklungsgeschichte des charakteristischen, leichten Gewebes, das nicht aus einem Kork- sondern einem Holzkambium entsteht und nach Goebel ein Aerenchym darstellt, das den unter dem Wasserspiegel befindlichen und deshalb unter Sauerstoffmangel leidenden Pflanzenteilen den Gasaustausch erleichtere.

$\mathrm{Ob}$ und wie weit diese Annahme ihre Begründung in der Ausbildung des dann vielleicht am besten als „Luftholz“ oder Aeroxylem zu bezeichnenden Gewebes hat, wird in der v. V. bei der Münchener philosophischen Fakultät eingereichten Dissertation ${ }^{1}$ ) einer eingehenderen Untersuchung unterworfen.

$\mathrm{Zu}$ diesem Zweck erfolgt zunächst eine genauere Schilderung der anatomischen Verhältnisse der betreffenden Hölzer: Herminiera Elaphroxylon, Aeschynomene indica, Aeschynomene sp.; Sesbania aegyptiaca; Erythrina crista galli, Erythrina indica, Erythrina palustris, ein den Erythrinen nahestehendes unbestimmtes Holz aus dem Institutsmuseum, Erythrina sp?; Pterocarpus Draco; Nyssa silvatica; Cavanillesia sp. und zwei Barigudas.

1) Die mit Zeichnungen und dem Literaturverzeichnis versehene Originalarbeit in Schreibmaschinenschrift ist im pflanzenphysiologischen Institut München einzusehen. 
Es erübrigt sich an diesem Orte ein näheres Eingehen auf die anatomische Struktur der Hölzer. Zusammenfassend sei nur das für die folgenden Untersuchungen Wichtigste gesagt:

Mit Ausnahme von Sesbania aegyptiaca und Erythrina sp.? ${ }^{1}$ ) ist den untersuchten Hölzern als Grundmasse ein leichtes, zartwandiges, weitlumiges Gewebe - eben das "Luftholz" - gemeinsam. Seine Zellwände sind äußerst schwach verholzt. Seine Zellen führen nirgends Inhalt. Bei den meisten Hölzern besitzen diese dachgiebelartig geneigte Querwände, die durch dichte, große, einfache Tüpfelung auffallen. Sie sind längs relativ wenig gestreckt und schließen bei der Mehrzahl der Hölzer lückenlos aneinander, so daß bei ihnen nur innerhalb des Mark-strahlgewebes kleine Interzellularräume auftreten. Eine Ausnahme hiervon bilden nur Erythrina indica und palustris und das unbestimmte Holz - bei dem es sich ganz augenscheinlich auch um ein der Gattung Erythrina zugehöriges Holz handelt -, wo auch im Luftholz vereinzelt feine Interzellularen auftreten. Ein reiches Interzellularsystem besitzen dagegen Cavanillesia und die Barigudahölzer.

Bei Pterocarpus Draco ist die dachgiebelartige Neigung der Querwände kaum mehr ausgeprägt - die Enden der schon mehr faserförmigen Zellen zeigen jedoch auch hier besonders reiche Tüpfelung und bei Nyssa silvatica zeigen sich die Zellen des Luftholzes typisch prosenchymatisch zugespitzt. Bei Cavanillesia und den Barigudas sind die Luftzellen unregelmäßig-polygonal. Das ganze Gewebe trägt den Charakter von Hollundermark. Mit Abnahme der regelmäßigen Gestaltung der Luftzellen geht eine Abnahme des gleichmäßig stockwerkartigen Aufbaues Hand in Hand, der bei den Aeschynomenen am ausgeprägtesten ist.

Die stärker verholzten Elemente, Gefäße und sklerenchymatische Binden, treten dem Luftholz gegenüber sehr stark zurück. Wo die sklerenchymatischen Zellreihen, wie v. a. bei den Aeschynomenen und Erythrinen, fast geschlossene Ringe bilden, liegt die Vermutung einer Jahrringbildung oder der Entstehung periodischer Zuwachszonen nahe; eine Entscheidung dieser Frage wäre jedoch nur bei Untersuchung und mehrjähriger Beobachtung der Pflanzen am natürlichen Standort möglich. Die durchweg hofgetüpfelten Gefäße sind bei Cavanillesia und den Barigudas sehr weit.

Erwähnt sei ferner das Vorkommen von „Wurzelanlagen“ bei den drei Aeschynomenearten, die auch eine rasche Bewurzelung bei Steck-

1) Dieses Holz scheint irrtümlich als Erythrina ausgezeichnet zu sein. 
lingsvermehrung ermöglichten. Ihre quer im Stamm verlaufenden Leitstränge weisen Ring- und Schraubengefäße auf, die übrigens bei den Aeschynomenen auch in Nachbarschaft der Markkrone vorkommen.

Kalziumoxalat führende Kristallkammerfasern wurden bei allen Aeschynomenen und Erythrinen, auch dem unbestimmten Holz, beobachtet.

Für Cavanillesia muß das fast völlige Fehlen stärker verholzter Elemente besonders hervorgehoben werden. Einzig die die Gefäße begleitenden Zellen sind etwas stärker verholzt, und nur ganz sporadisch findet man eine einzelne Holzfaser zwischen den Zellen der Grundmasse. Etwas zahlreicher sind die sklerenchymatischen Elemente bei den Barigudahölzern, welche im übrigen völlig der Cavanillesia entsprechen. Anßer einzelnen Holzfasern treten hier manchmal mehr oder weniger ausgeprägte Gruppen kurzer gekammerter Sklerenchymfasern auf.

Während Sesbania aegyptiaca und die Erythrina sp.? wegen ihres abweichenden Baues aus der Reihe der "Schwimmhölzer" von vornherein ausgeschaltet werden müssen, seien hier gleich noch ergänzend einige Hölzer angeführt, die nach früheren Untersuchungen zu dieser Gruppe gerechnet werden müssen. Solereder nennt noch: Aeschynomene americana L., Aesch. aspera L., Aesch. brasiliana D. C., Aesch. falcata D. C., Aesch. hispida Willd. und Aesch. hispidula. Außerdem gehören nach Vogelsberger noch Geissaspis cristata W. et. A. und tenella Bth. sowie Soemmeringia semperflorens hierher.

Leider liegen im allgemeinen keine Angaben darüber vor, ob diese Pflanzen auch im lebenden Zustand in den Zellen ihrer Grundmasse nur Luft führen. Nur für Aesch. sensitiva bringt Strasburger die Angabe, daß Schenk festgestellt habe, daß sie auch unter natürlichen Verhältnissen nur Luft in diesen Zellen enthalte. Ich fand dies am Gewächshausmaterial von Herminiera und Aesch. indica bestätigt. Diese Beobachtungen stützen die Annahme eines der Durchlüftung dienenden Gewebes in dieser Grundmasse ganz wesentlich.

Die Annahme eines Aeroxylem ist, wie erwähnt, schon deshalb naheliegend, weil die meisten dieser Pflanzen an feuchten, sumpfigen Flußufern gedeihen, ein äußerst rasches Wachstum zeigen und die unter Wasser befindlichen Stammteile an Sauerstoffmangel leiden mögen.

Dies gilt für die Aeschynomenen, Erythrinen und Pterocarpus Draco. Für Nyssa silvatica hingegen wird außer dem Vorkommen „an den Rändern von swamps“ noch das Auftreten in den mit Hochwald bedeckten Abhängen der Alleghany-Region angegeben, so daß bei ihr die Notwendigkeit eines Durchlüftungsgewebes nicht ohne weiteres 
einleuchtet. Es wäre interessant, Pflanzen beider Standorte nebeneinander zu untersuchen, um zu beobachten, ob und wie die Verschiedenheit desselben sich geltend macht.

Am unklarsten liegt die Sache bei Cavanillesia und den Barigudas ${ }^{1}$ ). Sie gehören zu den Charakterpflanzen der Catingas Südamerikas und werden ihres tonnenförmig angeschwollenen Stammes wegen auch als „Faßbäume" bezeichnet. Das Klima dieser Gegenden ist sehr trocken, ja es kommt, nach Goebel, vor, „daß ein unter Umständen 2 Jahre vergehen, bis diesen Teilen des Landes so viel Feuchtigkeit zugeführt wird, daß wieder Belaubung eintritt.“ Die tonnenförmige Anschwellung der Barigudas schiebt er auf die Entwicklung des Markes, welches als Wasserbehälter diene. Leider hat er selbst keinen Stamm untersuchen können. Die gleiche Anschauung vertritt Schimper, der aber auch nicht angibt, ob dieselbe durch Beobachtungen bestätigt ist. Auch Martius erwähnt das mächtig entwickelte Mark und das weiche Holz von Cavanillesia, jedoch ohne Angaben über Wasserführung innerhalb der Stammanschwellung. An sich liegt die Vermutung sehr nahe, in der Anschwellung einen Wasserspeicher zu erblicken, durch dessen Vorhandensein der Baum die lange Trockenzeit unbeschadet überstehen und sich trotz ihrer zu mächtiger Größe entwickeln könne. Auf die Trockenheit des Standorts und das dadurch entstehende Bedürfnis einer schneller, ausgiebigen Wasserleitung weisen ja jedenfalls die sehr weiten Gefäße hin. Leider lagen keine Angaben vor, ob die Holzproben dem angeschwollenen Achsenstück entnommen seien und wenn, ob der äußeren Zone desselben oder der Marknähe.

Es ist wohl denkbar, daß die untersuchten Stücke verschiedenen Zonen des Baumes entnommen sind und daß entweder im äußeren, rindenwärts gelegcnen Teil oder aber in der unverdickten Achse die sklerenchymatis. ion Elemcnte zahlreìcher sind, während sie gegen das Mark zu auf Kosten deı iockeren, dünnwandigen Grundmasse fast ganz verschwinden. Andererseits besteht die Möglichkeit, daß es sich um zwei Arten handele. Letzteres erscheint deshalb weniger wahrscheinlich, weil man sich schwer vorstellen kann, daß ein bis zu $20 \mathrm{~m}$ hoher Baum keine nennenswerten sklerenchymatischen Elemente besitzen soll. Es würden dann die relativ wenig verdickten Gefäße das einzige Stützorgan bilden.

Ob das weitlichtige Gewebe bei Cavanillesia tatsächlich ein Wasserspeicherorgan darstellt oder ob sich auch hier nur luftführende Zellen

1) „Bariguda“ ist der Eingeborenenname für die „Faßbäume“, die der Gattung Cavenillesia angehören. 
finden, deren Vorhandensein selbstverständlich nicht als eine Art „Anpassung" gedeutet werden dürfte, läßt sich nur an der lebenden Pflanze selbst entscheiden. Übrigens würde das ausgedehnte Interzellularsystem der Barigudas allein schon für genügende Durchlüftung sorgen.

Das Fehlen bzw. das äußerst geringe Auftreten von Interzellularen bei den anderen Hölzern spricht dagegen für die Deutung des beschriebenen Gewebes als Aerenchym; das Aeroxylem scheint den Mangel an Interzellularen ersetzen zu müssen.

Ferner spricht für diese biologische Deutung die äußerst geringe Verholzung und die sehr reiche Tüpfelung der Zellwände, die besonders im vertikalen Sinn einen raschen Gasaustausch gestattet. Dieses zeigt sich auch an den Versuchen, die über die Durchgangsgeschwindigkeit der Hölzer für Luft ausgeführt wurden. Leider gelang es nicht, die Gefäße völlig zu verstopfen, so daß der interessanteste Versuch über die Durchlaßgeschwindigkeit der Grundmasse der Hölzer für Gase unterbleiben mußte. So wurden nur allgemein vergleichende Versuche an den Schwimmhölzern und an einheimischen Hölzern gemacht. Dabei ergab sich für die „Lufthölzer" ein Maximalüberdruck von $3,2 \mathrm{~cm} \mathrm{Hg}$, für unsere Laubbäume ein Minimum von $5 \mathrm{~cm} \mathrm{Hg}$ und ein Maximum von $12,5 \mathrm{~cm} \mathrm{Hg}$, während bei Taxus sogar ein Druck von $30,5 \mathrm{~cm} \mathrm{Hg}$ nötig war, um am oberen Ende des dem kurzen Schenkel einer U-Röhre aufgesetzten Stammstückchens Luftblasen austreten zu lassen.

Die Schließhäute besonders der Querwandtüpfel des Luftholzes sind so fein, daß Goebel seinerzeit vermutete, sie möchten aufgelöst sein, da sie mikroskopisch nicht mit Sicherheit zu erkennen waren. Doch gelang es mir einigemale an sehr günstigen, mit Rutheniumrot gefärbten Schnitten mit Hilfe der Ölimmersion mit einiger Sicherheit Schließhäute zu beobachten. Um einwandfrei über das Fehlen oder Vorhandensein der Schließhäute entscheiden zu können, wandte ich außerdem zunächst noch eine schon von Goebel beschriebene Methode an: Das Durchsaugen von Suspensionen durch das Holz. Im Gegensatz zu Goebel, der ein Vordringen der Körnchen einer Karminsuspension bis in die 15. Zellage beobachtet hatte, setzten sich bei meinen Versuchen die Suspensionspartikelchen meist an der ersten Querwand $a b$, selten erst in der 2. und 3. Etage. Ebenso verlief der Versuch bei Anwendung einer Suspension von Berliner Blau. Das weitere Vordringen bei Goebels Versuch läßt sich daher wohl nur aus einem auf $\mathrm{zu}$ hohen Druck hin erfolgten Zerreißen der feinen Schließhäute erklären, nicht aber aus einem Fehlen derselben. Da bei Vorhandensein von Tüpfelschließhäuten auch ein Vordringen von 
Kolloiden nicht anzunehmen ist, wurde auch ein mineralisches „Suspensionskolloid" verwendet und zwar kolloides Arsensulfür. Um ein bei Gegenwart mineralischer Bestandteile leicht erfolgendes Ausfällen des Sulfürs $\mathrm{zu}$ verhindern, wurde erst längere Zeit destilliertes Wasser durchgesaugt, dann erst das Kolloid. Dieses wurde dann durch Einsaugen verdünnter Salzsäure zur Ausfällung gebracht. An Hand von Schnitten erkannte man dann, daß die Gefäße weit hinauf ausgefälltes Sulfür enthielten, während es in den „Luftzellen“ nur in der angeschnittenen Zellreihe $\mathrm{zu}$ beobachten war. Es sammelte sich hier, wie die Suspensionskörnchen, an den geneigten Querwänden an. Schnitt man das untere Ende des Stammstückes - ca. $1 \mathrm{~cm}$ - ab und saugte erneut Salzsäure hindurch, so mußte, war das Kolloid weiter hinauf vorgedrungen, auch hier eine Ausfällung erfolgen. Dies war nicht der Fall.

Um sicher zu gehen, daß nicht irgendwelche mineralischen Bestandteile, die vielleicht trotz des Auswaschens zurückgeblieben waren, gleich zu Beginn das Sulfür ausfällten und dieses durch Verstopfen der Poren ein weiteres Vordringen verhindere, mußte bei einem zweiten Versuch zwischen das Durchsaugen des Kolloids und der Salzsäure eine mikroskopische Untersuchung eingeschaltet werden. In der Tat setzten sich noch vor der Ausfällung mit Salzsäure Arsensulfürflöckchen an der Zellwand ab.

Dafür gibt es zwei Erklärungen: Entweder erfolgte tatsächlich durch das Vorhandensein mineralischer Bestandteile eine sofortige Ausfällung von Arsensulfür, das die Poren verstopfte. Dagegen spricht die Beobachtung, daß die Salzsäure niemals in der zweiten Zellreihe eine Ausfällung hervorrief. Eine Verstopfung der Poren wird aber schwerlich so momentan und vollkommen erfolgen, daß das Kolloid nirgends mehr in höhere Zellagen vordringen könnte. Ferner ist nicht anzunehmen, daß die Menge mineralischer Reagentien schon in der ersten Zellreihe trotz des Auswaschens so groß ist, daß sie eine völlige Ausflockung bewirken könnte.

Zweitens aber ließe sich der Tatbestand darauf zurückführen, daß Schließhäute vorhanden sind, die die ultramikroskopischen Teilchen des Suspensionskolloids nicht hindurchlassen. Diese häufen sich dann an den Querwänden an und ballen sich zu mikroskopisch sichtbar werdenden Flöckchen zusammen oder bewirken durch Änderung der Konzentration eine Ausfällung. Diese erfolgte übrigens auch bei Anwendung von Glyzerin als „Schutzkolloid.“

Daher ist mit größter Wahrscheinlichkeit anzunehmen, daß es Schließhäute sind, die ein Vordringen des Kolloids verhindern. Es 
400 Elisabeth Ewald, Beiträge zur Kenntnis der sogenannten Schwimmhölzer.

sprechen also sowohl die Experimente wie die mikroskopische Untersuchung für das Vorhandensein von Tüpfelschließhäuten bei Herminiera und Aeschynomene ${ }^{1}$ ). Dieselben sind nur ganz auffallend fein, was sicher den Gasaustausch von Zelle zu Zelle sehr erleichtert.

In anatomischer Hinsicht ist es also die schwache Verholzung, das Fehlen oder die geringe Ausbildung des Interzellularsystems, der Reichtum an oft großen und zartwandigen Tüpfeln, in physiologischer Beziehung die Luftführung der Zellen, die leichte Durchlüftungsmöglichkeit und der feuchte Standort, die bei den beschriebenen Hölzern mit größter Wahrscheinlichkeit in dem charakteristischen Gewebe ein Aerenchym vermuten lassen. Nur bei Cavanillesia, den Barigudas und vielleicht auch Nyssa silvatica ist eine solche Deutung nicht anzunehmen.

Sicher ist, daß mit Ausbildung eines solchen Aeroxylems keine „Anpassung" der Pflanzen an schwimmende Lebensweise vorliegt. Der Ausdruck „Schwimmhölzer" ist durchaus irreführend. Das Gewebe entsteht erst sekundär aus dem Holzkambium, rein zufällig oder aus einem Bedürfnis der Pflanze heraus, weswegen aber noch nicht einzusehen ist, warum gerade hier das sonst so verbreitete Interzellularsystem zur Schaffung einer Binnenatmosphäre verlassen und durch das Aeroxylem ersetzt wird.

Meinem verehrten Lehrer, Herrn Geheimrat von Goebel, möchte ich auch an dieser Stelle für die Anregung zur vorliegenden Arbeit und das Interesse, das er mir bei ihrer Ausführung stets entgegenbrachte, meinen aufrichtigsten Dank aussprechen.

1) Bei den übrigen Hölzern sind dieselben mikroskopisch unzweifelhaft $\mathrm{zu}$ erkennen. 